\title{
Internet of MIMO Things: UAV-assisted Wireless-powered Networks for Future Smart Cities
}

\author{
Oktay Cetinkaya, Member, IEEE, Domenico Balsamo, and Geoff V. Merrett, Senior Member, IEEE
}

\begin{abstract}
Widespread and pervasive IoT adoption is threatened by finite-capacity batteries of wireless devices. To mitigate this issue, energy harvesting (EH) and wireless power transfer (WPT), in addition to energy-efficient communication techniques, have been widely explored. Although these efforts achieved longevity to some extent, ever-evolving IoT services seek fully autonomous things without energy constraints. To meet this demand and relieve the ongoing networking challenges, we propose a new concept called the Internet of MIMO Things (IoMIMO). The IoMIMO envisions a self-sufficient architecture that adopts only single- and double-hop energy and data transitions to enable efficient energy sharing and reduced data traffic in networks. In particular, single-hops are performed by hybrid access points (HAPs), while relaying via double-hops are actuated by unmanned aerial vehicles (UAVs). The HAPs will handle multiple-input and multiple-output (MIMO) of energy and data, and coordinate their transitions between the network components in a concurrent and automated manner. Benefiting from the recent advances in multi-source EH, WPT, and UAVs, the IoMIMO can fulfill Smart City services without being limited by energy and networking challenges. Device types specialized for the IoMIMO, and their operation modes are evaluated in a simple network scenario to clearly explain the principles and the potential benefits of the envisioned concept. Future research directions are also identified to ease the realization of such a next-generation networking architecture.
\end{abstract}

Index Terms-Energy Harvesting, Wireless Power Transfer, SWIPT, Wireless-powered Networks, Green Communications, UAV Networking, Internet of Things, Smart Cities.

\section{INTRODUCTION}

Internet of Things (IoT) facilitates remote monitoring and control of any medium with wireless devices (e.g., sensor nodes) deployed in large numbers [1]. Despite promising capabilities, IoT networks suffer from limited device batteries. Randomness in depletion due to varying duties of devices envisioned by the IoT paradigm makes battery replenishment difficult. The proliferation of IoT services, therefore, necessitates novel procedures to assure perpetual operations. Here, energy-efficient communication techniques and energy harvesting (EH) mechanisms [2] stand out, while fostering a well-known paradigm called Green Communications [3].

The authors are with the Centre for IoT and Pervasive Systems in the School of Electronics and Computer Science, University of Southampton, Southampton SO17 1BJ, UK. e-mail: \{oc1y18, db2a12, gvm\}@ecs.soton.ac.uk.

D. Balsamo is also with the School of Electrical and Electronic Engineering, Newcastle University, Newcastle upon Tyne, UK. e-mail: ndb163@ncl.ac.uk.

This work was supported by the UK Engineering and Physical Sciences Research Council (EPSRC) under EP/P010164/1. There is no data associated with this paper.
Motivated by enabling potential, EH emerged as an alternative to the batteries, whereby the energy required for devices is opportunistically generated from ambient resources. A specialized version of it, namely wireless power transfer (WPT) [4], facilitates device energization at a distance and often achieves higher flexibility and lower maintenance costs [5], [6]. Both of these technologies have been extensively studied to put into practice for sustaining IoT services across diverse domains, notably in Smart Cities. On the other hand, unmanned aerial vehicles (UAVs) can be useful in alleviating the energy constraints by providing on-demand energy. Unification of $\mathrm{EH}$ and WPT, in support of UAVs [7], has therefore great potential to enhance the performance of networks, especially in remote areas of cities, lacking infrastructure and human support.

Considering this, we propose a self-sufficient network architecture that achieves efficient energy sharing and reduced data traffic via single- and double-hop energy and data transitions. The single-hops are performed by Hybrid energy and information Access Points (HAPs) deployed throughout the network, while the double-hops are fulfilled by flying mobile agents, i.e., UAVs. The HAPs harvest energy from multiple resources to remotely energize multiple components with multiple antennae, where the energy shared in the network is gathered solely by domain-specific EH mechanisms [8]. They also sustain half- and full-duplex communications to simultaneously collect multiple sensor data and ensure coordination between network components, besides offloading the measurements to the cloud via Internet Protocols (IPs). Thus, the HAPs refer to a Multiple-Input and Multiple-Output (MIMO) system in regard to energy and data transitions. This IP-enabled MIMO system, therefore, defines a new network paradigm called the Internet of MIMO Things (IoMIMO).

The IoMIMO offers a unique platform for multi-disciplinary efforts by embodying several technologies, such as sensing, information and communication, UAV networking, EH, WPT, and simultaneous wireless information and power transfer (SWIPT). To ensure faster, more reliable, and high-efficient energy and data transitions, The IoMIMO adopts five distinct operation modes for seven readily available devices, which are tailored to respond to the needs of emerging Smart City services. With these fully autonomous and selfsustaining devices, energy-aware networks promoting Green Communications will be achieved. Especially with the UAVs, lack of mobility and responsiveness in the IoT context will be mitigated, pioneering a new and enhanced networking experience. 


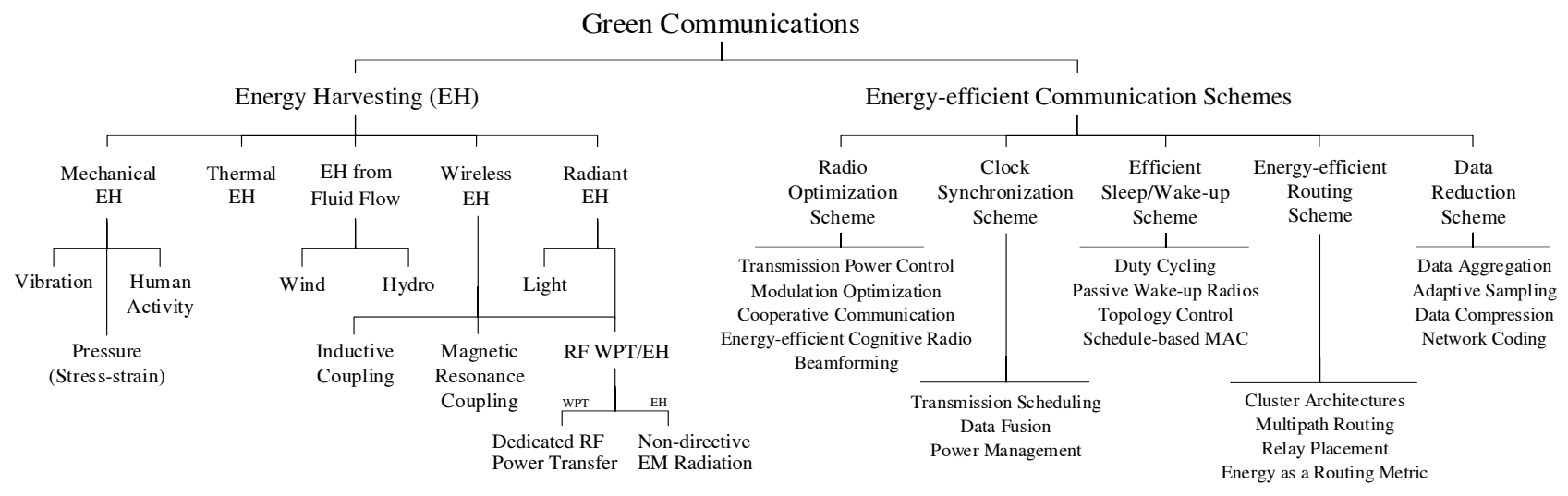

Fig. 1: Taxonomy of Green Communications [9], [10].

The remainder of this paper is organized as follows. Firstly, the principles of our proposal considering Green Communications and the underlying technologies are disclosed in Sec. II. This is followed by the operation overview, explaining the taxonomy and attributes of the devices to be employed. An example network scenario unveiling the individual relations between the components is also provided in Sec. III. Then, multi-dimensional objectives of the UAVs, in accordance with the IoMIMO-specific challenges and requirements, are explained in Sec. IV. Finally, possible application domains of the IoMIMO in the Smart City context are elaborated in Sec. V.

\section{Enabling Technologies for Green COMmunications in SMART Cities}

Green Communications promote the acquisition of an auxiliary energy source or effective utilization of the existing one(s) to prolong network lifetimes. Lengthened operation duration can be achieved with competent sleep/wake-up schemes, i.e., aggressive duty-cycling and topology control, in which the radio is periodically turned off (for power saving) and on (for idle-listening or transmission). The idle-listening power can be highly reduced by wake-up receivers, enabling intermittent operations. There are some other techniques (e.g. transmission power control, modulation optimization) tailored to optimize radio activity and improve clock synchronization [9].

Energy-efficient routing schemes, such as multi-path routing, can minimize congestions and data collisions in networks. Data reduction schemes, i.e., data compression/aggregation and adaptive sampling, can decrease the amount of sampled data while keeping the accuracy acceptable. However, all these techniques aim to extend the lifetime of wireless devices, where the networking aspects are mostly disregarded.

Contrary to existing efforts focusing on only one or a few enablers of Green Communications, this work aims to embody the whole branches of EH, shown in Fig. 1, with the support of energy-efficient communication techniques and emerging technologies. In such a way, networking challenges can be addressed by self-sufficient operations that are adaptive to domain-specific variations. Key constituents of the envisioned network architecture are detailed in the following subsections.

\section{A. Energy Harvesting}

EH is the exploitation of any resource to create electricity, where an energy storage is replenished without human supervision [2]. Thus, it is highly beneficial for especially widespread and hard-to-reach networks. EH is usually categorized into five groups, i.e., mechanical, thermal, fluid flow, radiant, and wireless $\mathrm{EH}$, features of each are briefly discussed below.

Solar power, which is based on the photovoltaic (PV) effect, is commonly used in rural sites. For indoor, however, specialized PV cells exploit artificial light propagation. Wind and hydro power, i.e., flow energy, is another alternative to operate devices in a self-sufficient fashion. Any mechanical movement (e.g. vibration, pressure variation) can be utilized thanks to piezoelectric materials. Thermoelectric generators can enable battery-less operation of wearable devices and Smart Building services by benefiting from temperature gradients. In urban areas, RF EH stands out due to wireless signals in abundance.

The power density of exploitable sources, however, is typically low and varies depending on harvester efficiency, deployment location, and many other uncontrollable factors. This innately promotes multi-source $\mathrm{EH}$, which enhances the energy output, enabling self-sufficient communications [11].

\section{B. Wireless Power Transfer}

WPT can be broadly classified into three categories: inductive coupling, magnetic resonance coupling, and RF power delivery [4]. In terms of accessible ranges, the first two refer to near-field, while the last stands for far-field energy transfer.

1) Inductive coupling: is based on magnetic-field induction between two coils, which ends up in energy transfer from one to the other [5]. It typically occurs within a range of a few centimeters due to the dramatic drop in induction effect over distance. Its efficiency depends on several factors, such as tightness of coupling and the quality factor of coils, which can reach up to $87 \%$ [4].

2) Magnetic resonance coupling: offers higher efficiency and wider coverage (up to meters) than inductive coupling at the cost of complexity and flexibility [4], [10].

3) $R F$ power delivery: is based on electromagnetic (EM) radiation, in which the RF signals emitted by an EM source are captured and rectified by a rectenna. Despite the low efficiency (typically $<40 \%$ ), it presents numerous advantages, e.g., small 
form factors and effective energy multi-casting [4]. It often requires devices to be in line-of-sight of the power source, which has to comply with the frequency-specific effective isotropic radiated power (EIRP) limits of the regulatory organizations, such as the Federal Communications Commission (FCC), imposed for human safety. For example, the maximum EIRP allowed on the $2.4 \mathrm{GHz}$ band is $36 \mathrm{dBm}(4 \mathrm{~W})$.

There are two types of RF power delivery, namely radiative and directive, categorized according to signal source characteristics. The former only needs a small antenna to capture waves propagating in any direction, while the latter requires precise alignment. Below, we further explain these methods.

- Radiative: Low-power devices can be potentially sustained by ambient RF signals, meaning that the energy acquisition may happen due to any source with omni-directional EM radiation. This method is especially useful for interference mitigation in densely-populated networks.

- Dedicated: Conveyance of considerably more power to longer distances is enabled by a controllable/directive source. A recent technique called SWIPT [6] allows the concurrent transfer of information and power on the same waveform, which improves bandwidth utilization efficiency.

Fig. 2 depicts the typical SWIPT architectures, namely separated, time switching (TS), power splitting (PS), and antenna switching (AS). This paper, however, elaborates on the most studied ones, i.e., TS and PS [5]. In TS, the receiver antenna alternately switches between $\mathrm{EH}$ and information decoding units, allowing them to individually utilize the received signals for certain durations: $\alpha \mathrm{T}$ and $(1-\alpha) \mathrm{T}$. In PS, the signal is divided into two separate streams, shared between $\mathrm{EH}$ and information decoding units, by a factor, $\rho$. TS typically has a simpler design and implementation compared to the PS; however, PS provides better trade-offs [10]. Depending on channel conditions, $\alpha$ and $\rho$ need to be dynamically adjusted.

Specific to application requirements, the above-explained technologies will be partially or fully utilized in the proposed architecture, building the future Smart City services.

\section{OPERATION OVERVIEW OF THE IOMIMO}

This section overviews the planned operation in a simple network scenario with regards to the technologies elaborated in Sec. II. To ensure efficient energy sharing and reduced data

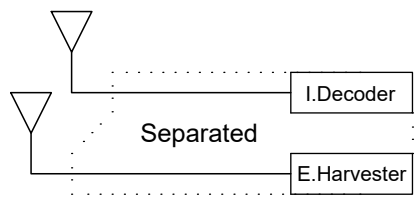

(a)

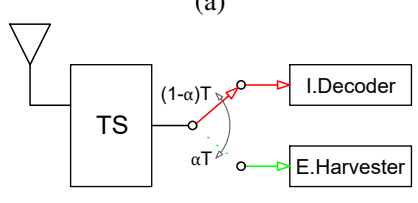

(b)

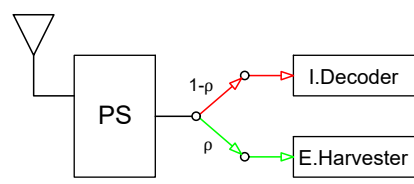

(c)

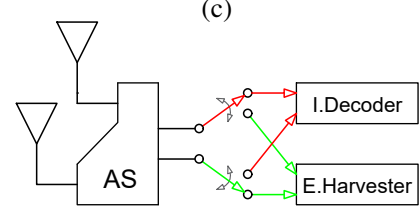

(d)
Fig. 2: SWIPT architectures, (a) Separated; (b) TS; (c) PS; (d) AS. traffic, the IoMIMO adopts five distinct operation modes for seven readily available devices. Firstly, the device types are described and classified, Fig. 3. Then, a simple network model, as in Fig. 4, is depicted. Finally, the operation overview, revealing the unique relations between the interoperating devices and the basics of the IoMIMO, is provided.

\section{A. Device Types \& Classification in the IoMIMO}

The proposed network architecture is mainly composed of Energy Providers (EPs), Data Gateways (DGs), and sensor nodes. The EPs exploit multiple sources to harvest energy, which is shared between network components by complying with FCC regulations. The DGs collect data from sensor nodes and create a gateway to the Internet via 4G LTE/5G-based communication technologies. The sensor nodes can be either wireless-powered or EH-operated with active or passive radios. The devices belonging to these categories are described below.

HAPs: are the backbone of the network, which operate as a co-located EP and DG. They handle multiple-input and -output of energy and data, and coordinate their flow between the network components in single- or double-hops. They have multi-antennae with beam-forming capabilities, granting spatial degrees of freedom in reaching longer distances without causing interference.

LETs: i.e., Local Energy Transmitters, are EPs with multisource EH capabilities. They deliver energy to the sensors via dedicated RF power transfer. They are also equipped with inductive coupling transmitter coils to enable opportunistic energization of the UAVs when needed.

LDTs: i.e., Local Data Transceivers, are DGs that collect data from sensor nodes to enable the monitoring of any medium or parameter in real-time. They divide the event space into Internet-enabled zones. Similar to HAPs and LETs, they also have multi-source EH capabilities to sustain their operations.
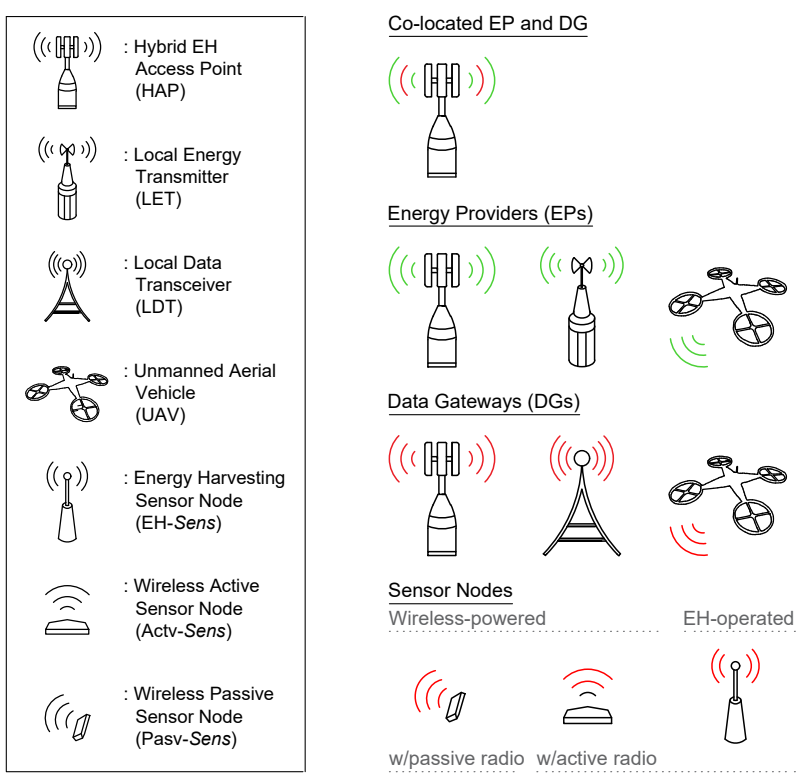

Fig. 3: Device types and classification in the IoMIMO. 


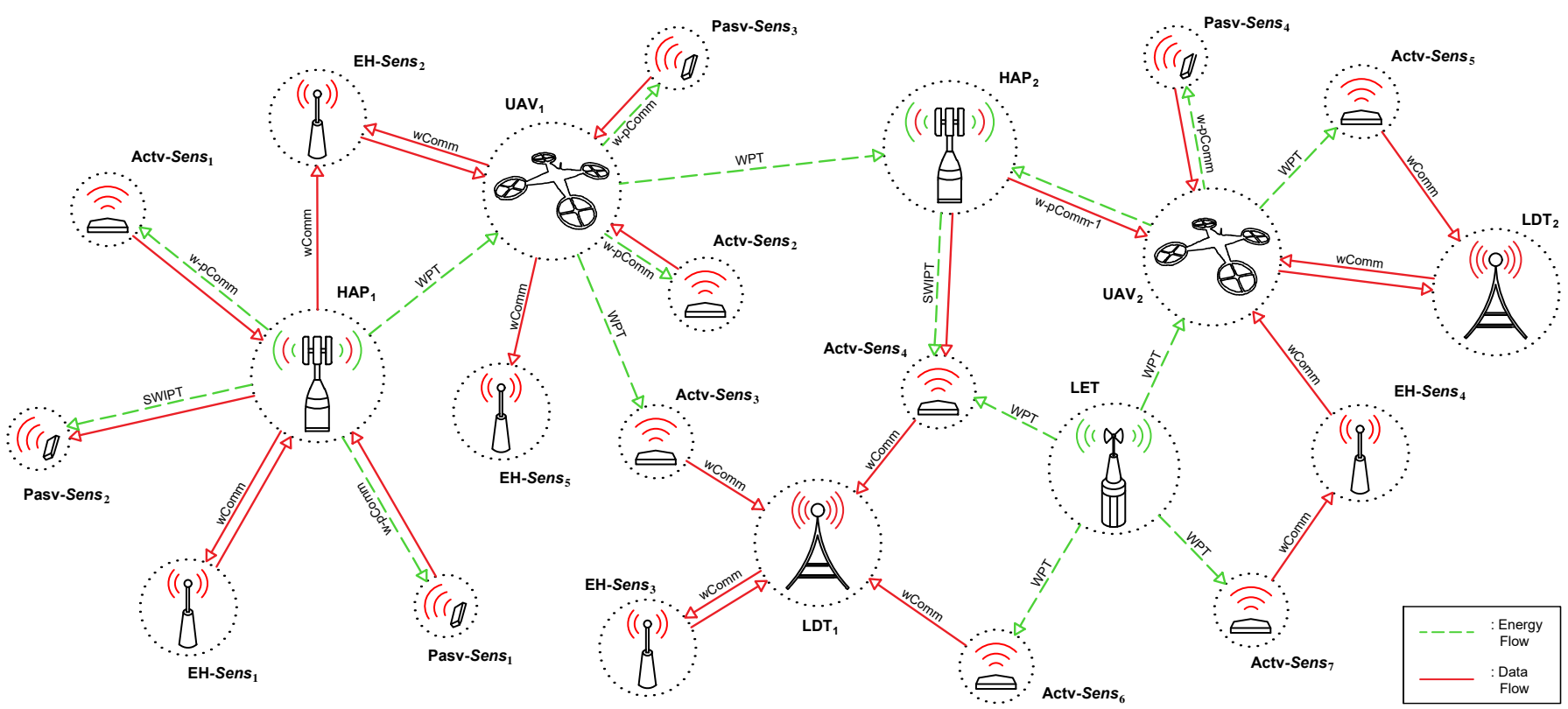

\begin{tabular}{|c|c|c|c|c|c|c|c|c|c|c|c|c|c|}
\hline 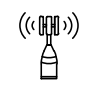 & $\begin{array}{l}\text { : Hybrid EH } \\
\text { Access Point } \\
\text { (HAP) }\end{array}$ & ((10p)) & $\begin{array}{l}\text { : Local Energy } \\
\text { Transmitter } \\
\text { (LET) }\end{array}$ & $\stackrel{((p)))}{A}$ & $\begin{array}{l}\text { : Local Data } \\
\text { Transceiver } \\
\text { (LDT) }\end{array}$ & $\theta$ & $\begin{array}{l}\text { : Unmanned Aerial } \\
\text { Vehicle } \\
\text { (UAV) }\end{array}$ & $((p))$ & $\begin{array}{l}\text { : Energy Harvesting } \\
\text { Sensor Node } \\
\text { (EH-Sens) }\end{array}$ & こ & $\begin{array}{l}\text { : Wireless Active } \\
\text { Sensor Node } \\
\text { (Actv-Sens) }\end{array}$ & (rod) & $\begin{array}{l}\text { : Wireless Passive } \\
\text { Sensor Node } \\
\text { (Pasv-Sens) }\end{array}$ \\
\hline
\end{tabular}

Fig. 4: An illustrative network model for the IoMIMO.

UAVs: operate as a mobile EP and DG. By drifting in three-dimensional (3D) space, they convey energy and data between network components, i.e., the relays of double-hop transitions. In such a way, they reduce the data traffic by obviating the need for complex and costly routing schemes.

EH-Sens: are the sensor nodes with active radios and EH capabilities. They are for mission-critical but delay-tolerant measurements. They are expected to deliver their readings to an LDT, a HAP, or a UAV within certain periods, duration of which are defined by the EH method.

Actv-Sens: are the sensor nodes with active radios. By using the energy transferred by EPs, they convey time-uncritical measurements to DGs, whereby their data become reachable at any-time and from anywhere. The Actv-Sens can also communicate with EH-Sens.

Pasv-Sens: are the sensor nodes with no active radios. They are the simplest devices in the network that perform sensing as long as they receive energy from an EP. The sensed parameters, mostly uncritical, are then transferred to the DGs via backscattering.

\section{B. Network Model for the IoMIMO}

Fig. 4 presents the envisioned network model, showing the energy and data flows between its components. The network enables efficient energy sharing and reduced data traffic with single- and double-hop transitions by adopting five distinct operation modes. These modes are defined in consideration of downlink and uplink transitions as follows:

WPT: power transfer in downlink (e.g., $\mathrm{HAP}_{1}$ to $\mathrm{UAV}_{1}$ ), SWIPT: SWIPT in downlink (e.g., $\mathrm{HAP}_{2}$ to Actv-Sens ${ }_{4}$ ),
wComm: i.e., wireless communications, information transfer in downlink and/or uplink (e.g., HAP ${ }_{1}$ to EH-Sens 2 ),

w-pComm: i.e., wireless-powered communications, power transfer in downlink and data retrieval in uplink (e.g., $\mathrm{HAP}_{1}$ to Pasv-Sens $1, \mathrm{HAP}_{1}$ to Actv-Sens ${ }_{1}$ ),

w-pComm ${ }^{-1}$ : i.e., inverse wireless-powered communications, data transfer (energy request) in downlink and power retrieval in uplink (e.g., $\mathrm{HAP}_{2}$ to $\mathrm{UAV}_{2}$ ).

A matrix, better illustrating the unique relations between the components and their designated operation modes, is provided in Table I. Several factors determine the interchange between modes, e.g., channel conditions, energy and data queues, duty cycles/reporting frequencies, pre-defined tasks, changing demands of network components, and instantaneous events. Regarding all these, the HAPs will schedule the operations and manage the alteration of modes so as to maximize the network performance.

TABLE I: Operations of the devices in the IoMIMO.

\begin{tabular}{|c|c|c|c|c|c|c|}
\cline { 3 - 7 } \multicolumn{2}{c|}{} & \multicolumn{6}{c|}{ Operation Modes } \\
\cline { 3 - 8 } \multicolumn{2}{c|}{} & WPT & SWIPT & w-pComm & w-pComm & wComm \\
\hline \multirow{4}{*}{} & HAP & $\checkmark$ & $\checkmark$ & $\checkmark$ & $\checkmark^{\dagger}$ & $\checkmark$ \\
\cline { 2 - 7 } & LET & $\checkmark$ & $x$ & $x$ & $\checkmark^{\dagger}$ & $\checkmark$ \\
\hline \multirow{2}{*}{} & LDT & $x$ & $x$ & $x$ & $\checkmark^{\dagger}$ & $\checkmark$ \\
\hline \multirow{2}{*}{} & UAV & $\checkmark / \circ$ & $x$ & $\checkmark$ & $\circ$ & $\checkmark$ \\
\cline { 2 - 7 } & EH-Sens & $x$ & $x$ & $x$ & $x$ & $\checkmark$ \\
\cline { 2 - 7 } & Actv-Sens & $\circ$ & $\circ$ & $\circ$ & $x$ & $\checkmark$ \\
\cline { 2 - 7 } & Pasv-Sens & $x$ & $\circ$ & $\circ$ & $x$ & $\checkmark$ \\
\hline
\end{tabular}

$\checkmark$ : compatible (master) $\|$ o: compatible (slave) $\| X$ : incompatible $\dagger$ : only if necessary 
The HAPs are also responsible for providing energy and data access to the sensor nodes and the UAVs. The UAVs can sustain all modes but SWIPT, as it may increase complexity in execution. Furthermore, their capabilities on WPT are relatively limited contrary to HAPs and LETs. In general, the UAVs operate on the $w$-pComm mode to collect sensor data by using the energy retrieved from HAPs and LETs in the WPT mode with inductive coupling. Furthermore, they can also trade energy between HAPs and LETs via $w$-p Comm $^{-1}$, with the help of $w C o m m$ and WPT modes.

In SWIPT mode, the Actv-Sens and Pasv-Sens carry out EH and information decoding tasks in downlink for concurrent data interpretation and device energization. As explained in Sec. II, accurate knowledge on channel state becomes highly crucial, since they need to switch between $\mathrm{EH}$ and information decoding units dynamically. Specific to the application, any method of SWIPT can be adopted.

In $w$-pComm mode, the Actv-Sens and Pasv-Sens intercept power in downlink and transmit their measurements in uplink. In similar, the EPs convey energy to a number of components with WPT in downlink, which will be utilized in the upcoming cycles. To exemplify, the Actv-Sens perform wComm in uplink by using this energy. Any low-cost protocol sustaining half- and full-duplex communications, defined by the ${ }^{\mathrm{w}} \mathrm{Comm}$ mode, can be employed in the network.

The IoMIMO, therefore, offers advanced flexibility and interconnectivity through diverse energy and data links, enabling autonomous and self-sufficient wireless services.

\section{Potential Enhancements offered by the IoMIMO}

As expected, WPT mode may cause interference at the DGs communicating in the same frequency band. This can be alleviated by allocating different bands for energy and data transitions. In that case, however, spectrum utilization efficiency will degrade since WPT often needs pseudo-random energy signals with non-negligible bandwidth usage. Here, the proposed EH-Sens come to the forefront for self-interference cancellation, enabling in-band wComm and WPT. Extracting energy from RF signals causing interference, i.e., self-energy recycling, not only contributes to the self-sufficiency vision of the IoMIMO but also devises a usage map of the EM spectrum. Regarding this map, cognitive radio functions can be employed to utilize the licensed/unlicensed bands even more efficiently. Furthermore, instead of adopting routing schemes increasing radio activity in the spectrum, the IoMIMO adopts UAVs for data collection from sensors. This will diminish the data traffic/congestion in networks, relieving the EM spectrum.

Unification of WPT and data access capabilities on HAPs, i.e., co-located EP and DG, eases the management of energy and data transitions in the network. This approach also reduces deployment, operation, and maintenance costs by concentric information collection, communication, and power transmission units. On the other hand, offloading of energy and data transitions to separate EPs and DGs, can mitigate the very well-known challenge of wireless-powered communications, namely doubly-near-far problem [6]. For example, a sensor (e.g., Actv-Sens 3 in Fig. 4) far from its EP, i.e., $\mathrm{UAV}_{1}$, retrieves lower energy in downlink due to path loss; however, needs more power in uplink to transmit its data contrary to another sensor (e.g., Actv-Sens 2 ) closer to that particular EP. This issue causes performance inequity as the nearby sensor will have higher throughput than the one in distance. With the IoMIMO, however, any sensor energized by an EP can transmit its data to a DG (to $\mathrm{LDT}_{1}$, instead of $\mathrm{UAV}_{1}$ ). Therefore, the doublynear-far problem can be resolved as wireless-powered sensors no longer need to transmit their data back to their EPs.

By proposing co-located and also separated DGs and EPs, not only enhanced flexibility and interconnection in a costeffective way is assured but also a solution to the ongoing issues on the technologies to be utilized is aimed so as to provide a next-generation networking experience.

\section{Multi-Dimensional Objectives of the UAVs IN THE CONTEXT OF THE IOMIMO}

The UAVs make the entire network more responsive to any circumstance, which is temporally and spatially nondeterministic in its nature. This grants a whole new set of benefits in terms of quality of service (QoS), e.g., reliability, pervasiveness, as well as enhanced flexibility and increased coverage probability. This section summarizes the key objectives of the UAVs in consideration of IoMIMO-specific challenges and requirements.

\section{A. Data Collection \& Handover}

The UAVs dynamically hover over the event area to support HAPs in keeping the network fully functioning. For example, in case of a communication failure, the UAV will be promptly sent to the darkened area to get in touch with the silent node(s) that the HAP can no longer hear. Although there are a number of reasons causing failures, the primary is energy inadequacy. To overcome this, the UAV can collect data with $w$-pComm from the node with insufficient energy and carry the obtained information to a DG. Alternatively, it can energize that node with WPT, helping it to increase its transmit power and reach the HAP again. In some cases, the node might be disabled, which necessitates replacement in the following cycles.

Scoping the ambient with the envisioned sensors in excessive numbers will result in extracting voluminous data. Here, the UAVs will work as auxiliary DGs by conveying the collected data to a HAP or an LDT, in consideration of energy levels (on both-hand sides), communication ranges, and prescheduled duties. These data will then be processed by scalable and reliable cloud services. This requires a dynamic decision algorithm for an effective handover mechanism [12].

\section{B. Energy Delivery}

The UAVs will retrieve energy from HAPs and LETs, and deliver the borrowed energy to Actv-Sens, Pasv-Sens, LDTs and even HAPs in need. They can also have the EH ability, whereby enhanced flexibility in energy sharing besides selfsufficiency is promoted [12]. Here, the timely update of the current energy levels of the devices is of utmost importance.

Since the energy budgets of the components are everchanging, a precise flight path determination is also required. 
Based on this, the UAV will actively change its route and the list of devices to be powered. Here, synchronization is highly crucial to enable high-efficient energy trading.

\section{Maintenance \& Deployment of Wireless Devices}

Throughout their lifetime, wireless devices are exposed to various factors (mostly ambient), which might damage their hardware or even destroy them. In such conditions, the UAVs will need to replace the non-functional, i.e., disabled, sensors to avoid any loss in the area being covered. The UAVs can also deliver additional sensors, LDTs, and LETs to cope with the ever-changing demands of the users/applications. The dynamic upgrade of the network will grant responsiveness and selfhealing as satisfying the architectural requirements of the IoT, i.e., scalability, flexibility, QoS, and continuity in operation [1]. Since the volume of sensor/LDT/LET inclusion may vary depending on the application, the network should be capable of handling massive device addition.

\section{Possible Application Areas of the Iomimo}

This section overviews the possible application domains of the IoMIMO, which are mostly related to remote and hard-toreach installations in Smart Cities.

\section{A. Increasing Agricultural Productivity}

Smart agriculture [3], [13] is one of the main target areas of the IoMIMO. Thanks to open and vast agricultural fields, the chance of harvesting energy from multiple sources and its amplitude will increase due to sunlight, air/water flows, and temperature gradients in abundance. Furthermore, the UAVs will hover around freely without any physical limitation.

Plant health monitoring for disease prediction, water level and irrigation control, bug invasion detection, fertilizer grade inspection, and even crop-spraying can be accomplished by the proposed sensors in support of the UAVs.

The bettered surveillance in agriculture with mechanized operations of the interconnected and self-sufficient devices offered by the IoMIMO will greatly increase productivity, so economic growth and development of the cities, which will enhance the quality of life and well-being of their citizens.

\section{B. Forest, Land \& Wildlife Monitoring}

The IoMIMO is expected to be used in securing forests, natural parks, and wildlife by promoting remote and perpetual observations against fire [13].

Thanks to the air-to-ground links facilitated by the UAVs, sensor data will be delivered to the DGs even in the most critical conditions, making the proposed network resilient to catastrophic events. The DGs notified with unreasonable changes in temperature and/or density of carbon gases will alert city officials to mobilize fire brigade.

The IoMIMO will also assure efficient utilization/deployment of the scarce number of sources/personnel by timely and accurate notifications with the UAVs drifting in the $3 \mathrm{D}$ space.

\section{Air \& Water Quality Monitoring}

The EH-Sens, Actv-Sens, and Pasv-Sens deployed in cities can measure the quality of air that the residents are exposed to. The data depicting pollution level will be conveyed to the city administrators over the DGs for taking protective measures, such as initiating purification processes and/or preventing vulnerable people, i.e., elders, patients, and children, from going outside until the air quality is bettered [14].

The IoMIMO will also perform pollution monitoring, air freshness control, and contaminator detection in water reservoirs/supplies to promote more resilient cities.

\section{Better Utilization of City Resources and Infrastructure}

Control of communication infrastructure and electrical power grid, structural health and noise monitoring, and traffic surveillance are some other services defined in the IoMIMO.

With the eased interconnection of cyber and physical worlds by the self-sufficient and autonomous devices of the IoMIMO, the cities will get even smarter and more livable. The efficient unification of IoT and key enabling technologies will escalate more people-centric and ambient-aware wireless services in Smart Cities, increasing the transparency between city officials and civilians. By providing alerts and insights, which are more relevant to residents, a better quality of life will be ensured.

\section{Vi. Conclusions and Future Research Directions}

This paper envisions a novel concept, adopting only singleand double-hop energy and data transitions to enable efficient energy sharing and reduced data traffic in networks.

The IoMIMO suggests the utilization of readily available devices and off-the-shelf products, and benefiting from the recent advancements in the key enabling technologies, such as multi-source EH, WPT, SWIPT, and UAV networking. With this agenda, seven different device types and five distinct operation modes were explained in a well-defined network scenario to clarify the potential benefits of the proposed architecture. To build these devices in a cost- and volume-effective way, mostly engineering efforts will be required. Constructive combination of multiple EH sources and their connection to WPT hardware, switching from inductive couplers to RF beam-formers, integration of efficient charge control mechanisms and antenna arrays will be some of the initial steps to be taken.

In addition to physical layer-related efforts, intelligent mechanisms will be essential to ensure the management of energy and data transitions between the network components. Furthermore, communication protocol(s) to be employed should have the self-configuration ability and offer modularity, enabling hassle-free modifications. The protocol stack should be revisited in consideration of IoMIMO-specific challenges. 3D channel modeling, flight path determination, efficient handover mechanisms will also be needed.

Unification of EH and WPT, in support of UAVs, will greatly enhance the Smart City services, especially in remote areas lacking infrastructure and human support. With the IoMIMO, the vision for IoT will be also achievable as the fundamental drawbacks, i.e., self-sufficiency, pervasiveness, and mobility, will be alleviated. 


\section{REFERENCES}

[1] I. Yaqoob et al. "Internet of Things Architecture: Recent Advances, Taxonomy, Requirements, and Open Challenges," IEEE Wireless Communications, vol. 24, no. 3, pp. 10-16, June 2017.

[2] M.-L. Ku et al. "Advances in Energy Harvesting Communications: Past, Present, and Future Challenges," IEEE Communications Surveys \& Tutorials, vol. 18, no. 2, pp. 384-1412, Nov. 2016.

[3] F. K. Shaikh et al. "Enabling Technologies for Green Internet of Things," IEEE Systems Journal, vol. 11, no. 2, pp. 983-994, June 2017.

[4] X. Lu et al. "Wireless Charging Technologies: Fundamentals, Standards, and Network Applications," IEEE Communications Surveys \& Tutorials, vol. 18, no. 2, pp. 1413-1452, Nov. 2016.

[5] S. Bi, C. K. Ho and R. Zhang. "Wireless Powered Communication: Opportunities and Challenges," IEEE Communications Magazine, vol. 53, no. 4, pp. 117-125, Apr. 2015.

[6] S. Bi et al. "Wireless Powered Communication Networks: An Overview," IEEE Wireless Communications, vol. 23, no. 2, pp. 10-18, Apr. 2016.

[7] O. Cetinkaya and G. V. Merrett, "Efficient Deployment of UAV-powered Sensors for Optimal Coverage and Connectivity," to appear in Proc. of IEEE Wireless Communications and Networking Conference, Apr. 2020.

[8] O. Cetinkaya et al. "Energy-Neutral Wireless-Powered Networks," IEEE Wireless Communications Letters, vol. 8, no. 5, pp. 1373-1376, Oct. 2019.

[9] T. Rault et al. "Energy Efficiency in Wireless Sensor Networks: A Topdown Survey," Computer Networks, vol. 67, pp. 104-122, July 2014.

[10] N. Zhao et al. "Exploiting Interference for Energy Harvesting: A Survey, Research Issues, and Challenges," IEEE Access vol. 5, pp. 10403-10421, May 2017.

[11] O. B. Akan et al. "Internet of Hybrid Energy Harvesting Things," IEEE Internet of Things Journal, vol. 5, no. 2, pp. 736-746, Apr. 2018.

[12] T. Long et al. "Energy Neutral Internet of Drones," IEEE Communications Magazine, vol. 56, no. 1, pp. 22-28, Jan. 2018.

[13] C. Perera et al. "Sensing as a Service Model for Smart Cities Supported by Internet of Things," Transactions on Emerging Telecommunications Technologies, vol. 25, no. 1, pp. 81-93, Sept. 2014.

[14] A. Zanella et al. "Internet of Things for Smart Cities," IEEE Internet of Things Journal, vol. 1, no. 1, pp. 22-32, Feb. 2014

[15] E. Ahmed et al. "Internet-of-Things-based Smart Environments: State of the Art, Taxonomy, and Open Research Challenges," IEEE Wireless Communications, vol. 23, no. 5, pp. 10-16, Oct. 2016.
Oktay Cetinkaya received his B.Eng. degrees (Hons.) with a double major in Electrical Engineering, and Electronics and Communication Engineering from Yildiz Technical University, Istanbul, Turkey, in 2013 and 2014, respectively. He then received his Ph.D. degree in Electrical and Electronics Engineering from Koc University, Istanbul, Turkey, in 2018. He is currently a Research Fellow at the University of Southampton. His research interests include energy harvesting-aided wireless-powered communications for the Internet of Things.

Domenico Balsamo received his B.Sc. and M.Sc. degrees in Electronic Engineering from the University of Modena and Reggio Emilia, Italy, in 2005 and 2008, respectively. He then received his Ph.D. degree in Computer Engineering from the University of Bologna, Italy, in 2015. He was appointed as a Lecturer in Embedded Systems Design and IoT at the Newcastle University in 2019. He was previously employed as a research fellow at the University of Southampton. His research broadly covers energy-efficient embedded systems and low-power pervasive computing, on which he published over 30 scientific papers in highly-respected journals and top-notch conference proceedings.

Geoff V. Merrett received the B.Eng. (Hons) and Ph.D. degrees from the University of Southampton, U.K., in 2004 and 2008, respectively. He currently holds a Personal Chair in Electronic and Software Systems at the School of Electronics and Computer Science, University of Southampton, U.K., where he is Head of the Centre for Internet of Things and Pervasive Systems. Prior to becoming a Professor in 2019, he was a Lecturer and Associate Professor at Southampton from 2009 and 2014 respectively. He is Technical Manager of the Arm-ECS Research Centre, an award winning collaboration between the university and Arm Research, Cambridge. His current research interests are in energy management of mobile/embedded systems and self-powered devices, and he has published over 200 journal and conference articles on these topics. Prof. Merrett was co-editor of the book "Many-Core Computing: Hardware and Software" (IET, 2019), General Chair of EWME 2016 and ENSsys 201315, and an Associate Editor for IET CDT and MDPI Sensors. He is a Member of the IET, and a Fellow of the HEA. 